\title{
Optimal sensor and actuator placement for structural health monitoring via an efficient convex cost-benefit optimization
}

\author{
Sergio Cantero-Chinchilla ${ }^{\mathrm{a}, \mathrm{d}, *}$, James L. Beck ${ }^{\mathrm{b}}$, Manuel Chiachío ${ }^{\mathrm{c}}$, Juan Chiachío ${ }^{\mathrm{c}}$, Dimitrios Chronopoulos $^{\mathrm{a}}$, Arthur \\ Jones $^{\mathrm{a}}$ \\ a Institute for Aerospace Technology \& The Composites Group, The University of Nottingham, NG7 2RD, United Kingdom \\ $b$ Department of Mechanical and Civil Engineering, California Institute of Technology, Pasadena, CA 91125, United States \\ c Dept. Structural Mechanics \& Hydraulics Engineering, University of Granada, 18001, Spain \\ d Aernnova Engineering Division S.A., Madrid, 28034, Spain
}

\section{Abstract}

The number and position of sensors and actuators are key decision variables that dictate the performance of any structural health monitoring system. This paper proposes choosing them optimally by using an objective function that combines a measure of parameter uncertainty, the expected information entropy, along with the cost of both sensors and actuators. The resulting optimization problem over discrete decision variables is computationally challenging, but here it is convexified by relaxing them into continuous variables, thus obtaining a significant reduction of the computational cost. The proposed approach is applied to ultrasonic guided-wave based inspection and is illustrated using two case studies with arbitrary geometries and different materials. The results demonstrate the high efficiency and accuracy of the convex optimization in trading-off uncertainty and cost in order to provide optimal sensor configurations in complex structures. As a key contribution, the proposed methodology allows us to include the actuators with the sensors in the optimization problem while still maintaining the efficiency of the minimization process. In the application to ultrasonic guided-waves, the optimal configurations lead to set-ups where the sensors and actuators are coincident in number and position.

Keywords: Optimal sensor configuration, Optimal actuator configuration, Entropy, Time of flight, Structural health monitoring, Guided waves

\section{Introduction}

Efficient and reliable condition-based maintenance requires an accurate and effective diagnosis provided by a suitable structural health monitoring (SHM) system. Such effectiveness depends heavily on the configuration (number and location) of sensors and actuators. Inaccurate and biased diagnosis in safety-critical structures may lead to erroneous actions such as false positive (also known as Type I error) or false negative (Type II error) alerts [1], which might lead to catastrophic economical and safety-related consequences. In order to provide a reliable, yet efficient, configuration

${ }^{*}$ Corresponding Author

Email address: Sergio. CanteroChinchilla1@nott ingham. ac. uk (Sergio Cantero-Chinchilla) 
for a SHM system, the selection of an optimal sensor and actuator configuration is desirable, that provides a balance between the performance of the SHM system and the related cost of sensors and actuators [2]. To this end, several approaches have been reported in the literature, which can be broadly categorized into two groups, namely approaches using the value of information [3, 4] and those using cost-benefit analysis [5, 6]. The first approach provides the sensor and actuator configuration that holds the best balance between cost and information gain of the SHM system. However, its main limitation lies in its computational cost, especially in complex real-life engineering applications, such as ultrasonic guided-wave based SHM [7]. Alternatively, the second one typically uses performance indexes and costrelated information associated to the layout of sensors of the SHM system, which are simultaneously maximized and minimized respectively in a multi-objective type of optimization algorithm. This approach, although less informative, is more generic and therefore it provides a higher flexibility to adapt to real-world engineering applications.

The optimal placement of sensor transducers within an ultrasonic guided-wave based SHM system has been addressed in the literature for both active and passive sensing diagnosis by maximizing the performance of the systems with specific indexes. One such index is the probability of detection, which is typically defined ad-hoc by quantifying different features such as the distance of the damage from the sensor [8] or the strain caused by an impact [9]. Another one is the area of coverage, which relies on geometrical and physical properties [10, 11], as well as the influence of defective sensors [12]. This approach has proven effective in maximizing the coverage of control points [13], assuming sensor locations as continuous variables [14], and considering different types of sensors [15]. The area of coverage has also been combined with numerical, experimental, and analytical information to address the sensor optimization in an efficient yet accurate manner [16]. Besides, wave propagation modeling approaches can be used directly for optimizing transducer locations, e.g. by selecting the areas with the largest wave amplitude [17]. Their main limitation, however, lies in the computational cost, which can be alleviated if approximation techniques are adopted such as the continuous wavelet transform [18]. However, their optimization procedure relies on combinatorial algorithms such as genetic algorithms [19, 20], simulated annealing [21, 22], or particle swarm optimization [23] and may, in general, give a suboptimal layout with no indication of its accuracy. Additionally, the optimal joint positioning of actuators and sensors has been studied in structural applications other than ultrasonic guided-wave based SHM by maximizing the controllability [24], observability, or spillover effect [25, 26]. Alternatively, convex optimization [27] techniques have been used to provide optimal configurations of sensors and/or actuators in an efficient manner [28-30] using an objective function that can be minimized using conventional algorithms for function minimization such as the interior point algorithm [31, 32], which is more efficient in terms of computational cost. However, none of them have considered the uncertainty present in the data and also in the guided-wave related model, thus limiting its robustness against noise or modeling uncertainty.

Bayesian approaches have been proposed to address the optimal sensor configuration by quantifying uncertainty using the Kullback-Leibler (KL) divergence between the prior and posterior distribution as a measurement of the information gain [33, 34], the Shannon information entropy of the posterior distribution as a measure of the uncertainty of posterior information [35, 37], and the mutual information [38, 39] between data and model parameters. In [39], the 
authors note that all three of these information-theoretic quantities give equivalent optimization problems for optimal location placement. More recently, a value of information based cost-benefit analysis for optimal sensor configuration in plate-like structures for ultrasonic guided-wave based SHM has been proposed in the literature [7].

To relieve this burden, an efficient method is proposed in this paper to obtain both the optimal number and location of sensors and actuators for SHM systems by minimizing a cost-benefit function in terms of Shannon's information entropy and a cost function. This approach extends the work previously developed in [38, 39], which addresses optimal placement of a fixed number of sensors by applying convexification and relaxation techniques to the entropy of the pre-posterior distribution, which is based on the model-predicted data rather than experimental data. By performing a cost-benefit optimization, we find the optimal number, as well as the optimal location of both sensors and actuators. Although the methodology presented here is generic and employable within any monitoring field involving actuators and sensors for obtaining information relating to a structural, chemical, or biological system, we illustrate it here using ultrasonic guided-wave based SHM. This application provides a challenging scenario involving the optimal configuration (number and location) of actuators and sensors over a two-dimensional space in isotropic and orthotropic materials. The chosen layout of the sensor and actuator grids and the area of possible damage occurrence are customizable, so that they can be adapted to any kind of structure and prior information. Nonetheless, the spatial resolution of each grid is limited by the size of these devices and their wiring. A high efficiency in obtaining the optimal configurations is observed in comparison with existing information-based approaches regardless of the complexity of the material, which enhances the use of this methodology in complex, real-world applications.

The remainder of this paper is organized as follows: Section 2 describes the proposed entropy-based convex optimization methodology for the configuration of actuators and sensors configuration; Section 3 illustrates the methodology through two case studies using two different plate-like structures; Section 4 provides a discussion of the impact of the proposed method on ultrasonic guided-wave based SHM. Finally, Section 5 provides concluding remarks.

\section{Methodology}

\subsection{Optimal placement for a given number of sensors and actuators}

Let $x_{i}^{j}(n, \boldsymbol{\theta}) \in \mathbb{R}$ denote a deterministic model prediction for parameters $\boldsymbol{\theta}$ of an arbitrary quantity of interest (QoI) at discrete time $n$ (e.g. time of flight of an ultrasonic wave or acceleration at a material point) at the $i$-th sensor, assuming that the input excitation is provided by the $j$-th actuator. We assume that $N$ observations will be available for this QoI, denoted by $\mathbf{D}_{N}=\left\{y_{i}^{j}(n) \in \mathbb{R}, n=1, \ldots, N\right\}$, when the sensors are installed. These observations could come from an experiment or simulation that is repeated $N$ times, or $n$ could be interpreted as an instant of time within a QoI time-history [35, 36]. The deterministic model prediction and the actual QoI are related by introducing a stochastic prediction-error term that accounts for the discrepancy between the model output and the actual observation [40], as follows:

$$
y_{i}^{j}(n)=x_{i}^{j}(n, \boldsymbol{\theta})+e_{i}^{j}(n)
$$


The uncertain prediction error is assumed to be modeled as a zero-mean Gaussian distribution with standard deviation $\sigma_{e} \in \mathbb{R}$ by applying the Principle of Maximum Information Entropy, which gives a distribution that has the largest uncertainty for specified first and second moments. Any other choice of distribution would have less uncertainty without any additional information [40, 41]. If the actual number of sensors and actuators installed are denoted by $N_{s}^{g}$ and $N_{a}^{g}$, respectively, then the predicted data for a specified sensor and actuator configuration is:

$$
p\left(\mathbf{D}_{N} \mid \boldsymbol{\theta}\right)=\prod_{j=1}^{N_{a}^{g}} \prod_{i=1}^{N_{s}^{g}} \prod_{n=1}^{N} p\left(y_{i}^{j}(n) \mid \boldsymbol{\theta}\right)
$$

where the prediction errors in Equation (1) are chosen to be independent stochastically, that is, if we know one prediction error, it provides no information about the others. Next, two grids of sensor and actuator positions of $N_{s}$ and $N_{a}$ points, respectively, are chosen to provide possible locations of these devices. The actual locations of the sensors and actuators are then selected by activating binary variables denoted by $\Psi_{s}^{(i)} \in\{0,1\}$ and $\Psi_{a}^{(j)} \in\{0,1\}$, respectively, where 0 and 1 correspond to the absence and presence of a sensor or actuator at the $i$-th and $j$-th locations of their respective sets of grid points. Thus, we can rewrite Equation (1) as:

$$
p\left(\mathbf{D}_{N} \mid \boldsymbol{\theta}\right)=\prod_{j=1}^{N_{a}} \Psi_{a}^{(j)} \prod_{i=1}^{N_{s}} \Psi_{s}^{(i)} \prod_{n=1}^{N} p\left(y_{i}^{j}(n) \mid \boldsymbol{\theta}\right)
$$

Assuming a large number of data points $N$ [35, 36], the posterior PDF of the model parameters $\boldsymbol{\theta}$ given the data $\mathbf{D}_{N}$ and a particular actuator and sensor configuration specified by $\boldsymbol{\Psi}_{a}$ and $\boldsymbol{\Psi}_{s}$ respectively, can be asymptotically approximated as [36]:

$$
p\left(\boldsymbol{\theta} \mid \Psi_{a}, \Psi_{s}, \mathbf{D}_{N}\right) \cong \frac{\left[\operatorname{det} Q\left(\boldsymbol{\Psi}_{a}, \boldsymbol{\Psi}_{s}, \boldsymbol{\theta}\right)\right]^{\frac{1}{2}}}{\left(2 \pi \hat{\sigma}_{e}\right)^{\frac{1}{2} N_{a} N_{s}}} \exp \left[-\frac{1}{2 \hat{\sigma}_{e}^{2}}(\boldsymbol{\theta}-\hat{\boldsymbol{\theta}})^{T} Q\left(\boldsymbol{\Psi}_{a}, \Psi_{s}, \boldsymbol{\theta}\right)(\boldsymbol{\theta}-\hat{\boldsymbol{\theta}})\right]
$$

where $\hat{\boldsymbol{\theta}}$ is the MAP (maximum a posteriori) value of the posterior distribution in Equation (4) and $Q\left(\Psi_{a}, \Psi_{s}, \boldsymbol{\theta}\right) \in \mathbb{R}^{N_{\boldsymbol{\theta}} \times N_{\boldsymbol{\theta}}}\left(N_{\boldsymbol{\theta}}\right.$ : the number of uncertain model parameters considered in the predictive deterministic model $\left.x_{i}^{j}(\boldsymbol{\theta})\right)$ is given by [42]:

$$
Q\left(\boldsymbol{\Psi}_{a}, \Psi_{s}, \boldsymbol{\theta}\right) \cong \sum_{j=1}^{N_{a}} \Psi_{a}^{(j)} \sum_{i=1}^{N_{s}} \Psi_{s}^{(i)}\left\{\sum_{n=1}^{N}\left(\nabla_{\boldsymbol{\theta}} x_{i}^{j}(\boldsymbol{\theta}) \nabla_{\boldsymbol{\theta}}^{T} x_{i}^{j}(\boldsymbol{\theta})\right)\right\}=\sum_{j=1}^{N_{a}} \Psi_{a}^{(j)} \sum_{i=1}^{N_{s}} \Psi_{s}^{(i)} P_{i}^{j}(\boldsymbol{\theta})
$$

where matrix $P_{i}^{j}(\boldsymbol{\theta})=\sum_{n=1}^{N} \nabla_{\boldsymbol{\theta}} x_{i}^{j}(\boldsymbol{\theta}) \nabla_{\boldsymbol{\theta}}^{T} x_{i}^{j}(\boldsymbol{\theta}) \in \mathbb{R}^{N_{\boldsymbol{\theta}} \times N_{\boldsymbol{\theta}}}$ and $\nabla_{\boldsymbol{\theta}} x_{i}^{j}(\boldsymbol{\theta})$ denotes the gradient vector $x_{i}^{j}(\boldsymbol{\theta})$. Notice that in Equation (4), the dependence on data $\mathbf{D}_{N}$ is only through the MAP values $\hat{\boldsymbol{\theta}}$.

When the optimal sensor and actuator configuration is being determined, data $\mathbf{D}_{N}$ will not be available, so a pre-posterior analysis is necessary where an expectation of the information entropy of the posterior in Equation (4) is taken, which is a measure of the posterior parameter uncertainty that also accounts for it being conditional on unknown 
future data that will be collected by the sensor network. The expectation of the posterior entropy is with respect to the data $\mathbf{D}_{N}$ as predicted probabilistically by the model in Equation (11).

An approximation was introduced in [36] that avoids such computationally demanding Monte Carlo simulation of samples for $\mathbf{D}_{N}$, as follows. Assuming large $N$, the MAP values $\hat{\boldsymbol{\theta}}$ of the model parameters are replaced by the nominal values $\boldsymbol{\theta}_{0}$ of the model parameters defined by their prior distribution $p\left(\boldsymbol{\theta}_{0}\right)$, which describes the designers' uncertainty about appropriate values of the model parameters. The entropy-based objective function can then be expressed as [36]:

$$
h\left(\boldsymbol{\Psi}_{a}, \boldsymbol{\Psi}_{s}\right)=-\mathbb{E}_{\boldsymbol{\theta}_{0}}\left[\log \operatorname{det} Q\left(\boldsymbol{\Psi}_{a}, \boldsymbol{\Psi}_{s}, \boldsymbol{\theta}_{0}\right)\right]
$$

The problem of optimal sensor and actuator placement now becomes the minimization of this pre-posterior measure of parameter uncertainty over discrete variables $\Psi_{s}^{(i)}$ and $\Psi_{a}^{(j)}$, where $\sum_{i=1}^{N_{s}} \Psi_{s}^{(i)}=N_{s}^{g}$ and $\sum_{i=1}^{N_{a}} \Psi_{a}^{(i)}=N_{a}^{g}$.

Next, based on the idea in [28, 38, 39], the variables $\Psi_{s}^{(i)}$ and $\Psi_{a}^{(j)}$ in Equation (6) are relaxed into continuous variables $z_{i}$ and $w_{j}$ in the interval $[0,1]$. Therefore, the function $Q$ now can be expressed as $Q\left(w, z, \boldsymbol{\theta}_{0}\right) \cong$ $\sum_{j=1}^{N_{a}} w_{j} \sum_{i=1}^{N_{s}} z_{i} P_{i}^{j}\left(\boldsymbol{\theta}_{0}\right)$ with $w_{j} \in[0,1]$ and $z_{i} \in[0,1]$. Equation 6 is then of the form of $f(X)=-\mathbb{E}_{X}[\log \operatorname{det}(X)]$, where $X$ is a positive semidefinite matrix that is linear in the continuous variables, and therefore the function is convex in the domain of these variables [27]. Thus, the original combinatorial optimization problem is transformed into a continuous convex optimization problem with respect to $z \in[0,1]^{N_{s}}$ and $w \in[0,1]^{N_{a}}$ that is readily solved numerically using classical convex minimization methods [28, 39] such as the interior-point algorithm:

$$
\begin{array}{cl}
\underset{w, z}{\operatorname{minimize}} & h(w, z)=-\mathbb{E}_{\boldsymbol{\theta}_{0}}\left[\log \operatorname{det} Q\left(w, z, \boldsymbol{\theta}_{0}\right)\right] \\
\text { subject to } & 0 \leq w_{j} \leq 1, j=1, \ldots, N_{a} \\
& 0 \leq z_{i} \leq 1, i=1, \ldots, N_{s} \\
& \sum_{j=1}^{N_{a}} w_{j}=N_{a}^{g} \\
& \sum_{i=1}^{N_{s}} z_{i}=N_{s}^{g}
\end{array}
$$

\footnotetext{
Note that $h\left(w^{*}, z^{*}\right)$, corresponding to the optimal values in Equation (7) for each $w_{j}^{*} \in[0,1]$ and $z_{i}^{*} \in[0,1]$, provides a lower bound of the minimum of $h\left(\Psi_{a}, \Psi_{s}\right)$ in Equation (6). If any $w_{j}^{*}$ or $z_{i}^{*}$ is not at its boundary value of 0 or 1 , then the solution to Equation (7) is not directly applicable in its continuous form in practice since the sensors or actuators can only be present or absent at each grid point, i.e., $w_{j}$ and $z_{i}$ need to be either 1 or 0 . In this case, the variables can be rounded to their closest binary value while ensuring that the constraints $\sum_{j=1}^{N_{a}} w_{j}=N_{a}^{g}$ and $\sum_{i=1}^{N_{s}} z_{i}=N_{s}^{g}$ are still satisfied. This gives a binary solution $\left(\mathbf{\Psi}_{a}^{*}, \Psi_{s}^{*}\right)$ where $h\left(\Psi_{a}^{*}, \Psi_{s}^{*}\right)$ is an upper bound of the exact minimum of $h\left(\boldsymbol{\Psi}_{a}, \boldsymbol{\Psi}_{s}\right)$. If these upper and lower bounds are close, then $\left(\boldsymbol{\Psi}_{a}^{*}, \boldsymbol{\Psi}_{s}^{*}\right)$ can be taken as a near-optimal placement solution.
} 


\subsection{Optimal actuator and sensor configuration: cost-benefit analysis}

Building on the problem of optimal actuator and sensor placement formulated in the previous sub-section, the optimal number of sensors and actuators may also be addressed by introducing a monotonically increasing dimensionless cost function $s(n):\left[1,\left(N_{a}+N_{s}\right)\right] \rightarrow[0,1]$ that quantifies the cost of adding $n$ devices into the SHM system. Note that the cost function includes both actuators and sensors in the same function, which is appropriate for our application of interest, ultrasonic guided-wave based SHM, where a piezoelectric (PZT) transducer can be used as either a receiver or emitter. However, $s(n)$ can be defined differently in other applications where sensors and actuators may have different costs. For efficient optimization, we choose variable $n$ to be real, lying in the interval $n \in\left[0,\left(N_{a}+N_{s}\right)\right]$. Since the objective function in Equation (7] is monotonically decreasing with respect to $n$ [43], i.e. the more devices that are added to the system, the less entropy (pre-posterior parameter uncertainty) is obtained, and $s(n)$ is monotonically increasing, we can define a new convex minimization problem as follows:

$$
\begin{array}{cl}
\underset{w, z, n}{\operatorname{minimize}} & h^{*}(w, z, n)=-\mathbb{E}_{\boldsymbol{\theta}_{0}}\left[\log \operatorname{det} Q\left(w, z, \boldsymbol{\theta}_{0}\right)\right]+\eta \cdot s(n) \\
\text { subject to } & 0 \leq w_{j} \leq 1, j=1, \ldots, N_{a} \\
& 0 \leq z_{i} \leq 1, i=1, \ldots, N_{s} \\
& \sum_{j=1}^{N_{a}} w_{j}+\sum_{i=1}^{N_{s}} z_{i}=n
\end{array}
$$

where $\eta>0$ is used to establish a particular trade-off between information gain and cost. We choose $s(n):\left[1,\left(N_{a}+\right.\right.$ $\left.\left.N_{s}\right)\right] \rightarrow[0,1]$ as a dimensionless cost function formed using interpolating monotonic cubic splines [44] due to their ease of implementation and versatility in mimicking almost any monotonically increasing cost function. The function $s(n)$ takes a value of 0 when there is the specified minimum number of sensors and actuators, and the value of 1 for the specified maximum number of them. As for the minimization in Equation (7), the convex optimization problem in Equation (8) may be addressed with standard convex minimization algorithms such as the interior-point algorithm. Hence, both the optimal placement and optimal number of actuators and sensors are simultaneously obtained in a very efficient, yet rigorous, manner.

\subsection{Bayesian damage localization using ultrasonic guided-waves}

Our presented approach for selecting the optimal sensor and actuator configuration is utilized here for ultrasonic guided-wave based SHM for damage localization in a plate-like structure [45]. The damage position is inferred using an ellipse-based model inverse problem and the ToF (time of flight) of the scattered wave from the damage to the sensors. The observations at each sensor are compared with the predictions of a ToF model, which in this case is defined as:

$$
x_{i}^{j}=\frac{\sqrt{\left(X_{d}-X_{a}^{j}\right)^{2}+\left(Y_{d}-Y_{a}^{j}\right)^{2}}}{V_{a-d}(f, \alpha)}+\frac{\sqrt{\left(X_{d}-X_{s}^{i}\right)^{2}+\left(Y_{d}-Y_{s}^{i}\right)^{2}}}{V_{d-s}(f, \alpha)}
$$




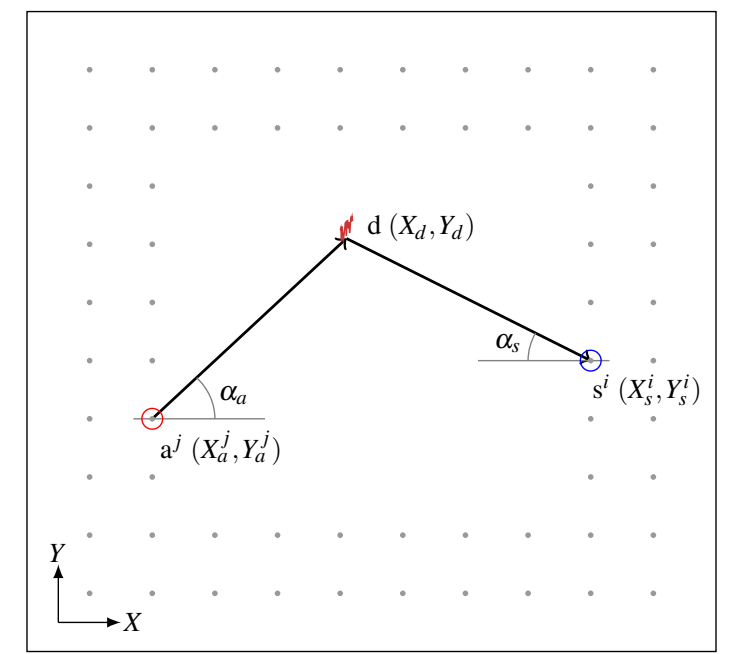

Figure 1: Graphical representation of the paths followed by the guided-waves emitted by the actuator $\mathrm{a}^{j}$, which interact with the damage $\mathrm{d}$ and are acquired at the sensor $\mathrm{s}^{i}$. The gray points represent the space of possible sensor/actuator positions.

where $\left(X_{d}, Y_{d}\right) \in \mathbb{R}^{2}$ are the coordinates of the damage position, $\left(X_{a}^{j}, Y_{a}^{j}\right) \in \mathbb{R}^{2}$ are the $j$-th actuator transducer coordinates, $\left(X_{s}^{i}, Y_{s}^{i}\right) \in \mathbb{R}^{2}$ are the coordinates of the $i$-th sensor transducer, and $V_{a-d}(f, \alpha)$ and $V_{d-s}(f, \alpha)$ are the wave propagation velocities of the actuator-damage and damage-sensor paths defined by the angle $\alpha$, respectively. Figure 1 depicts the geometrical characteristics of the paths from an arbitrary actuator to a sensor (selected from the possible sensor/actuator positions), passing through the damage position. In general, the velocities depend on the frequency of excitation $f$, the wave mode under investigation, and the direction of the path with respect to the material orientation. Assuming that the situation involves an orthotropic material, such as a composite laminate, these velocities can be approximated by modeling the velocity profile with respect to the angle for a given frequency. Here, the velocity is assumed to be distributed elliptically in space as in the case of angle-ply laminates, as follows [46]:

$$
V(f, \alpha)=\sqrt{V_{x}^{2}+V_{y}^{2}}=\sqrt{(a \cdot \cos (\gamma))^{2}+(b \cdot \sin (\gamma))^{2}}
$$

where the parameter angle $\gamma$ relates to the physical angle $\alpha[46]$ as follows:

$$
\gamma=\arctan \left(\frac{a}{b} \tan (\alpha)\right) ;\left\{\alpha_{a}=\arctan \left(\frac{Y_{d}-Y_{a}^{j}}{X_{d}-X_{a}^{j}}\right), \alpha_{s}=\arctan \left(\frac{Y_{s}^{i}-Y_{d}}{X_{s}^{i}-X_{d}}\right)\right\}
$$

where $\alpha_{a}$ and $\alpha_{s}$ denote the geometrical angle formed by the actuator-damage and damage-sensor paths, respectively, and $a$ and $b$ denote the two main axes of the velocity ellipse. Therefore, the set of uncertain parameters of the ToF model for orthotropic materials are the damage coordinates in addition to $a$ and $b$, so $\boldsymbol{\theta}=\left\{X_{d}, Y_{d}, a, b\right\}$. Alternatively, the set of parameters can be simplified if an isotropic structure (e.g. an aluminum plate) is considered. Then, the set 
of parameters would be $\left.\boldsymbol{\theta}=\left\{X_{d}, Y_{d}, V\right\}\right]^{1}$

\section{Case studies}

In this section, the optimal actuator and sensor configuration methodology is applied to plate-like structures with irregular, but realistic, geometries motivated by the shape of wing skin panels of standard commercial aircraft, as suggested in Figure 2
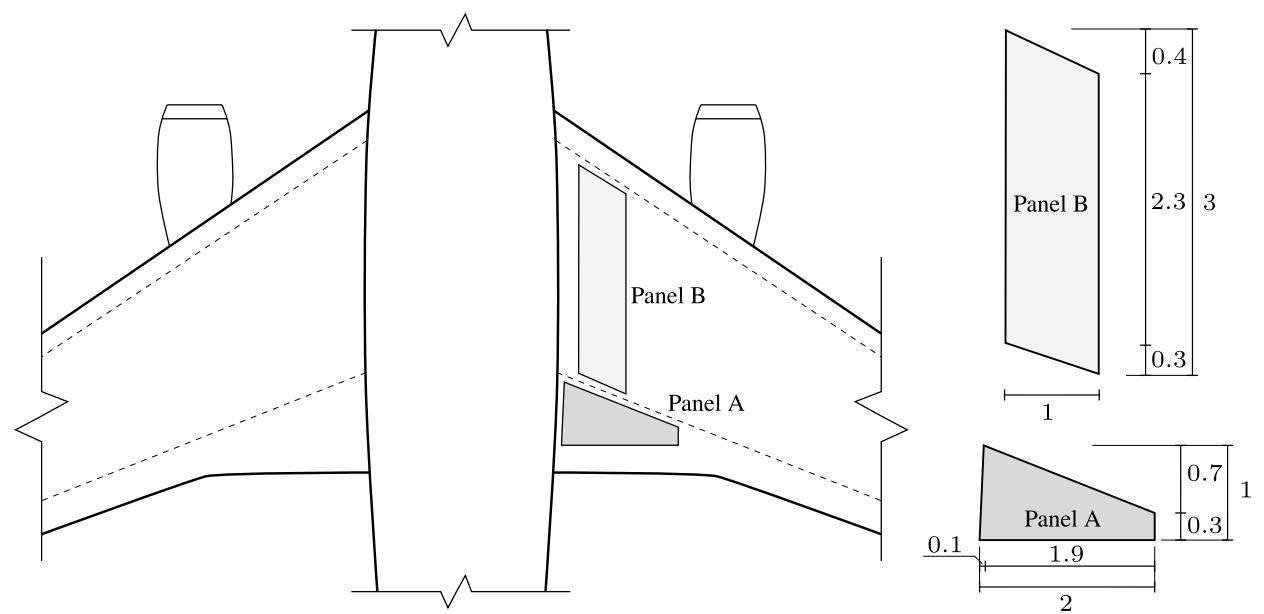

Figure 2: Top view of the central part of standard aircraft. Two typical skin panel geometries (A and B) with irregular geometries are highlighted in the right wing. Units expressed in meters.

\subsection{Description of structures and definition of problem}

Two thin-walled structures, named panels A and B, as depicted in Figure 2, are considered in this case study. Additionally, it is assumed that there exist two possible panels for each geometry A and B, denoted as panels A1, A2, B1, and B2, where the number refers to different materials. Panels A1 and B1 are made of aluminum alloy 2024-T351 with $2 \mathrm{~mm}$. On the other hand, panels A2 and B2 are made of a quasi-isotropic T800-M913 carbon fiber laminate with $\left[-45 / 90_{2} / 45 / 0\right]_{s}$ stacking sequence and $1.5 \mathrm{~mm}$ thickness. The mechanical properties of both materials are shown in Table 1

The guided-wave mode assumed for health monitoring of the aluminum sheets (panels A1 and B1) is the $\mathrm{A}_{0}$ mode at $300 \mathrm{kHz}$, whose group velocity is $3000 \mathrm{~m} / \mathrm{s}$. In case of the composite plates (panels A2 and B2), the $\mathrm{S}_{0}$ mode is chosen at $150 \mathrm{kHz}$, whose group velocity is angle-dependent following an elliptical profile defined by its major axis $a=7549 \mathrm{~m} / \mathrm{s}$ and its minor axis $b=6030 \mathrm{~m} / \mathrm{s}$, as shown in Figure 3a The wave propagation velocities for both aluminum and composite materials are related to their mechanical properties and obtained by computing their dispersion curves at different angles using the Dispersion Calculator [47]. Note that the only information needed to

\footnotetext{
${ }^{1}$ Note that both velocity terms are the same under the assumption of isotropic materials and damage concentrated within a bounded region, i.e. $V=V_{a-d}=V_{d-s}$.
} 
Table 1: Mechanical properties of both the aluminum structure and one layer of the composite material.

\begin{tabular}{lcccccc}
\hline Composite & Longitudinal stiffness & Transverse stiffness & Shear stiffness & Poisson's ratio & Poisson's ratio & Density \\
T800-M913 & $\mathrm{E}_{11}[\mathrm{GPa}]$ & $\mathrm{E}_{22}[\mathrm{GPa}]$ & $\mathrm{G}_{12}[\mathrm{GPa}]$ & $v_{12}[-]$ & $v_{23}[-]$ & $\rho\left[\mathrm{kg} / \mathrm{m}^{3}\right]$ \\
& 152.14 & 6.64 & 4.20 & 0.25 & 0.54 & 1550 \\
\hline Aluminum & Young's modulus & Poisson's ratio & Density & & & \\
$2024-\mathrm{T} 351$ & $\mathrm{E}[\mathrm{GPa}]$ & $v[-]$ & $\rho\left[\mathrm{kg} / \mathrm{m}^{3}\right]$ & & & \\
& 73.1 & 0.33 & 2780 & & \\
\hline
\end{tabular}

address the ToF-based actuator and sensor optimization is the wave propagation velocity, either as a constant value for isotropic materials, or angle-dependent in case of orthotropic materials.

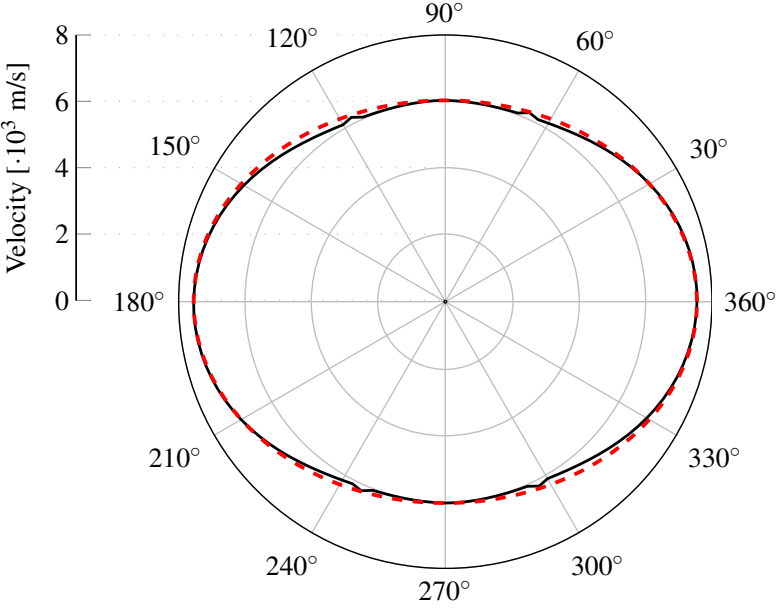

(a) Group velocity $\left(\mathrm{S}_{0}\right.$ mode) profile in the composite laminate.

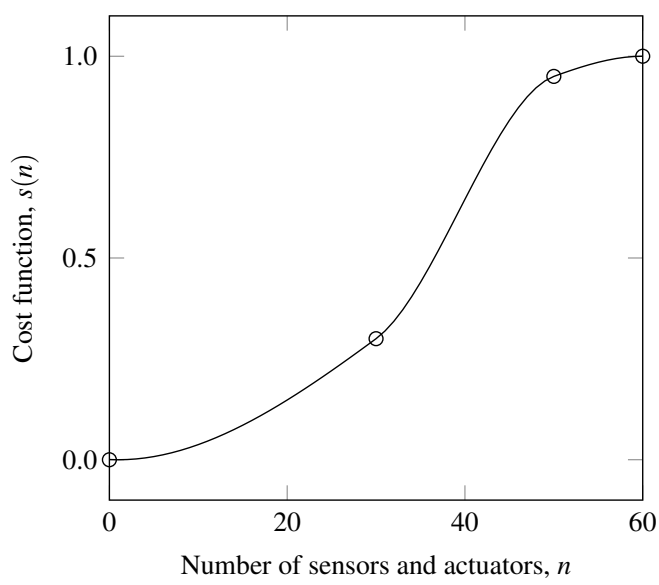

(b) Cost function used in the design of the SHM system.

Figure 3: Data used in the optimal design of the SHM system. In panel (a), angular dependence of the group velocity of the $\mathrm{S}_{0}$ mode at $150 \mathrm{kHz}$ (solid line) and its approximation by an ellipse with major axis $a=7549 \mathrm{~m} / \mathrm{s}$ and minor axis $b=6030 \mathrm{~m} / \mathrm{s}$ (dashed line). In panel (b), the cost function $s(n)$ used for the optimization of the number of sensors and actuators made by interpolating cubic splines between a number fixed points, represented by circles.

A cost function $s(n)$ is arbitarily defined in order to address the proposed combined optimization of the number and positions of PZT sensors and actuators (recall Eq. (8)) in the form of interpolating cubic splines with intermediate points that defined such a function, as shown in Figure $3 b$ The interpolating points are represented as circles in Figure $3 \mathrm{~b}$ and are as follows: $(0,0),(30,0.3),(50,0.95),(60,1)$, in pairs of $(n, s(n))$ where $n$ is the number of transducers. Here, the same cost per unit is used for both sensors and actuators, arising from the nature of guidedwave based SHM, which uses PZT transducers for emitting and receiving ultrasonic signals. Additionally, the trade-off between information gain and cost, dictated by $\eta$ in Equation $(8)$, is chosen to be $\eta=|h(w, z)|$ for the case studies presented hereinafter. 


\subsection{Optimal sensor and actuator configuration: panel A}

\subsubsection{Aluminum panel Al}

The results for the isotropic plate (panel A1) are obtained using a uniform distribution of possible damage occurrence inside the gray area represented in Figure 4a The prior uncertainty for the wave propagation velocity is quantified by a Gaussian PDF, $V \sim \mathscr{N}\left(3000,40^{2}\right)$ in [m/s] units. Then, 500 samples drawn from the prior distribution of the model parameters $\theta=\left\{X_{d}, Y_{d}, V\right\}$ are used in the optimization, along with the previously proposed cost function $s(n)$ in Figure $3 \mathrm{~b}$ Results from Figure $4 \mathrm{a}$ show that the optimal locations for the sensors and actuators are the same, generally at the corners, with the same optimal number of $\sum_{j=1}^{N_{a}} w_{j}=\sum_{i=1}^{N_{s}} z_{i}=6.30$ for each device type (i.e. $n=12.60$, as in Eq. (8)). Note that the total number of sensors and actuators is a real number since the decision variables $\left(w_{j}\right.$ and $\left.z_{i}\right)$ are continuous in the interval $[0,1]$. Note also that the results show only the sensor and actuator positions with $w_{j} \geq 0.2$ and $z_{i} \geq 0.2$ to better identify the optimal positions. These thresholds make the sum of decision variables $\sum_{j} w_{j}=4.726$ and $\sum_{i} z_{i}=4.722$ for actuators and sensors, respectively, which are different from the optimal numbers of sensors and actuators given above as they consider all the values below the previous threshold.

In the left corners, both the upper and bottom ones, the results provide two sensors and actuators placed next to each other. This behavior can be explained under the assumption of the stochastic independence of the data acquired by the sensors, which causes a sensor clustering effect. In this case, the optimal sensor and actuator configuration provides an objective function value (recall Eq. (8p) of $h^{*}(w, z, n)=55.0237$. However, such optimal configuration cannot be applied in practice, and therefore, a Boolean approximation is selected by rounding off the number of sensors and actuators (e.g. $6.30 \rightarrow 6$ ) for each device type, and selecting the six positions with higher $w_{j}$ and $z_{i}$ (see Table 2). This Boolean approximation provides an objective function value of $h^{*}\left(\Psi_{a}, \Psi_{s}, n\right)=55.0587$, which represents a remarkably close approximation to the optimal convex configuration. The coordinates of the positions above the specified threshold and the corresponding Boolean layout are given in Table 2 .

Table 2: Sensors and actuators above the specified threshold of $w_{j} \geq 0.2$ and $z_{i} \geq 0.2$ along with their corresponding coordinates and the approximate Boolean solution for the panel A1.

\begin{tabular}{lcccccc}
\hline & \multicolumn{2}{c}{ PZT coordinates } & \multicolumn{2}{c}{ Relaxed solution } & \multicolumn{2}{c}{ Binary solution } \\
PZT No. & $X[\mathrm{~m}]$ & $Y[\mathrm{~m}]$ & $w[-]$ & $z[-]$ & $\Psi_{a}$ & $\Psi_{s}$ \\
\hline 1 & -0.900 & -0.450 & 0.984 & 0.984 & 1 & 1 \\
2 & -0.800 & -0.450 & 0.489 & 0.483 & 1 & 1 \\
3 & 0.900 & -0.450 & 0.970 & 0.970 & 1 & 1 \\
4 & 0.900 & -0.200 & 0.715 & 0.723 & 1 & 1 \\
5 & -0.830 & 0.350 & 0.986 & 0.986 & 1 & 1 \\
6 & -0.825 & 0.400 & 0.582 & 0.576 & 1 & 1 \\
\hline
\end{tabular}

\subsubsection{Composite panel A2}

In the case of the composite panel A2, the same number of samples and prior distribution of the damage coordinates $\left(X_{d}, Y_{d}\right)$ used for the previous case (panel A1) are applied. The prior information of the parameters that define the elliptical model of the wave propagation velocity, $a$ and $b$, are distributed using Gaussian PDFs as follows: 


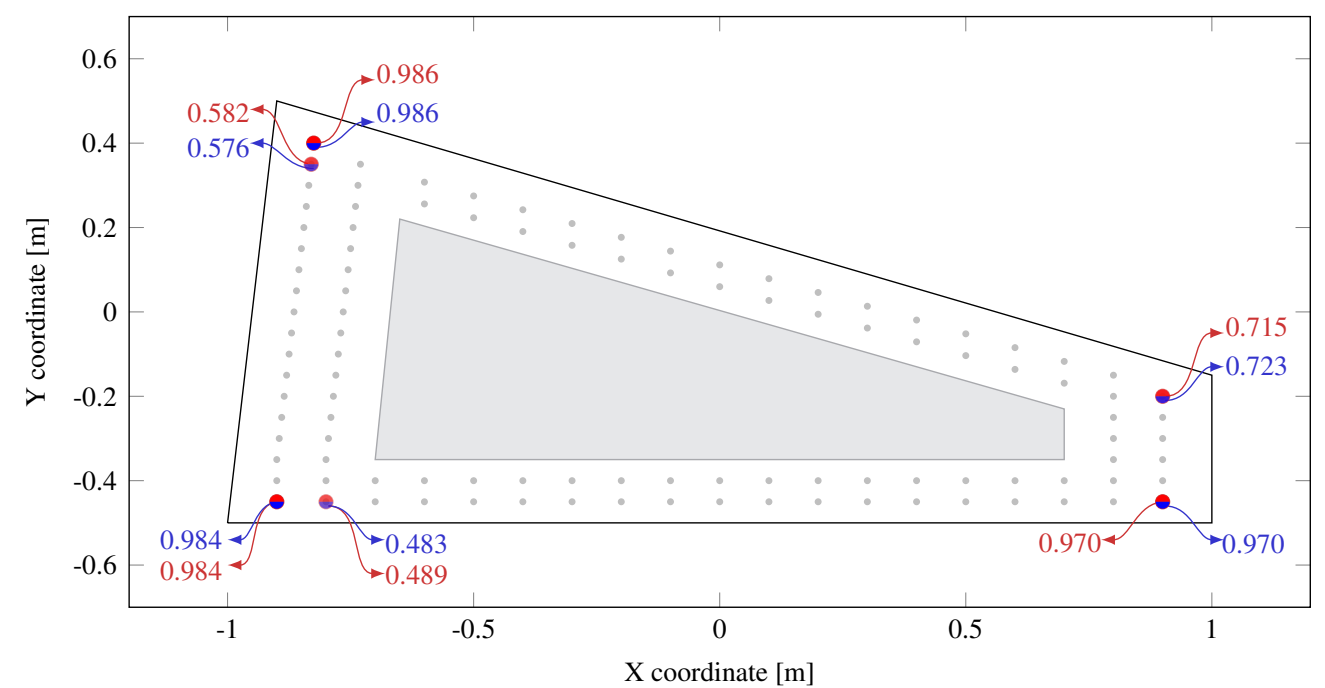

(a) Optimal actuator and sensor configuration for panel A1.

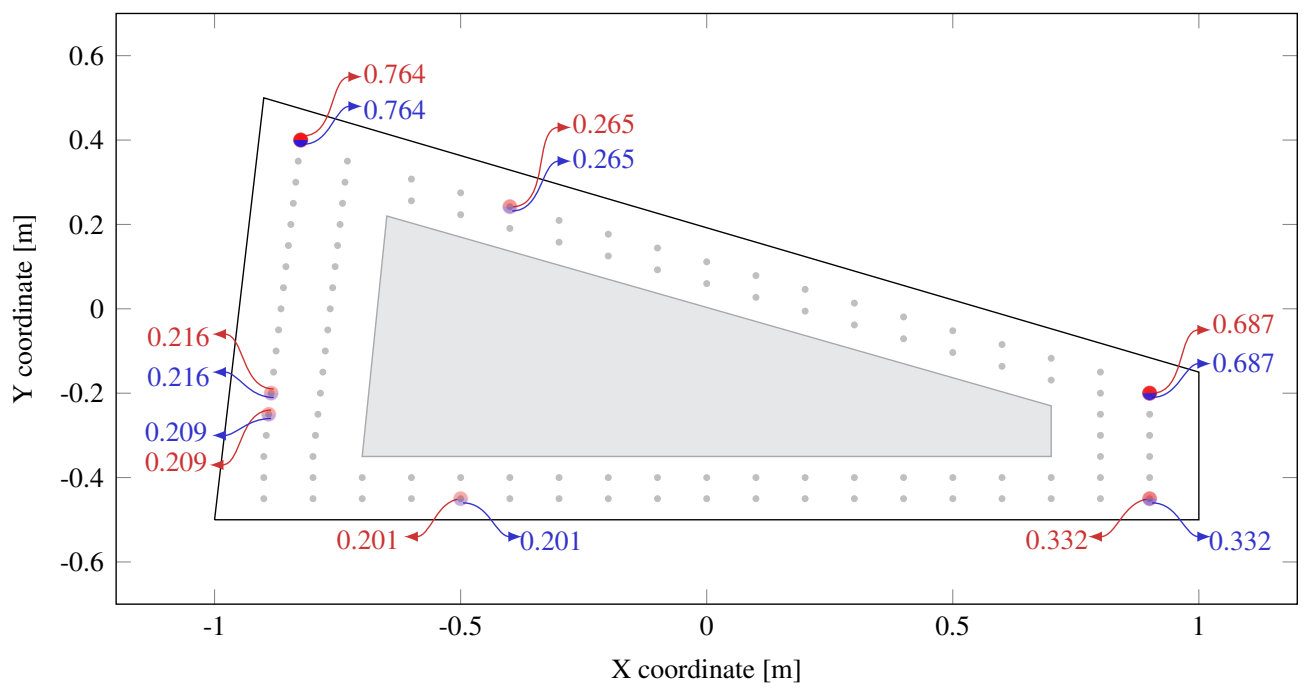

(b) Optimal actuator and sensor configuration for panel A2.

Figure 4: Optimal actuator and sensor configuration for the panel geometry A assuming different materials and prior distributions of the damage occurrence. (a) depicts the case for aluminum alloy 2024-T351 and a uniform prior over the gray inner polygon, and (b) shows the same geometry and prior uncertainty of potential damage occurrence in case of the composite laminate of layup $\left[-45 / 90_{2} / 45 / 0\right]_{s}$. Red and blue numbers represent the value of actuator and sensor decision variables $\left(w_{j}\right.$ and $\left.z_{i}\right)$, respectively.

$a \sim \mathscr{N}\left(6030,40^{2}\right)$ and $b \sim \mathscr{N}\left(7549,40^{2}\right)$, with both expressed in [m/s] units. Figure $4 \mathrm{~b}$ shows the optimal actuator and sensor configuration using the $s(n)$ defined in Figure $3 \mathrm{~b}$, which gives an equal optimal number for sensors and actuators of 5.33. Note that the transducers are distributed differently from those in the isotropic case, appearing at intermediate zones of the structure. The angular-dependent wave propagation profile (see Figure 3a) drives the optimal positioning of the transducers, hence highlighting the importance of carrying out optimal sensor and actuator configuration studies for structures with different materials, even if they share the same geometry. The seven sensors that appear in Figure $4 \mathrm{~b}$ are listed in Table 3 considering again the threshold values $w_{j} \geq 0.2$ and $z_{i} \geq 0.2$, and they 
lead to an objective function evaluation of $h^{*}(w, z, n)=99.6435$, which is a lower bound. Given the stochastic nature of the objective function and that several PZTs have similar $w$ and $z$ values, the practical solution using a Boolean approximation is addressed by exploring the combinations of PZT 3 and 6 (i.e. the ones with highest $w$ and $z$ values) with the rest of transducers listed in Table 3 The best sub-optimal Boolean approximation corresponds to the six sensors and actuators specified in Table 3 and results in $h^{*}\left(\Psi_{a}, \Psi_{s}, n\right)=100.2577$. Note also in Figure $4 \mathrm{~b}$ that PZT 4 and 5 are close to each other and that with this approximation one of them is dropped, hence avoiding sensor clustering.

Table 3: Sensors and actuators above the specified threshold of $w_{j} \geq 0.2$ and $z_{i} \geq 0.2$ along with their corresponding coordinates and the approximate Boolean solution for panel A2.

\begin{tabular}{lcccccc}
\hline & \multicolumn{2}{c}{ PZT coordinates } & \multicolumn{2}{c}{ Relaxed solution } & \multicolumn{2}{c}{ Binary solution } \\
PZT No. & $X[\mathrm{~m}]$ & $Y[\mathrm{~m}]$ & $w[-]$ & $z[-]$ & $\Psi_{a}$ & $\Psi_{s}$ \\
\hline 1 & -0.500 & -0.450 & 0.201 & 0.201 & 1 & 1 \\
2 & 0.900 & -0.450 & 0.332 & 0.332 & 1 & 1 \\
3 & 0.900 & -0.200 & 0.687 & 0.687 & 1 & 1 \\
4 & -0.890 & -0.240 & 0.209 & 0.209 & 0 & 0 \\
5 & -0.885 & -0.190 & 0.216 & 0.216 & 1 & 1 \\
6 & -0.825 & 0.410 & 0.764 & 0.764 & 1 & 1 \\
7 & -0.400 & 0.242 & 0.265 & 0.265 & 1 & 1 \\
\hline
\end{tabular}

\subsection{Optimal sensor and actuator configuration: panel B}

\subsubsection{Aluminum panel B1}

The results for the isotropic plate with the geometry B that are shown in Figure 5a are obtained in this case using a prior damage distribution of possible occurrence over the gray polygon with two concentrated areas. Thus, the a priori information of the $X_{d}$ coordinate is represented as a bimodal Gaussian distribution consisting of the weighted summation of two Gaussian PDFs, $X_{d} \sim\left\{1 / 2 \mathscr{N}\left(-0.6,0.2^{2}\right)+1 / 2 \mathscr{N}\left(0.6,0.2^{2}\right)\right\}$ with units expressed in meters, while the $Y_{d}$ is uniformly distributed, both within the bounded area represented in Figure 5a Furthermore, the wave propagation velocity $V$ is equally distributed as specified in Section 3.2.1 using the same number of prior samples to evaluate the objective function. As observed from the results shown in Figure 5 a the sensors and actuators above the previously specified thresholds $\left(w_{j} \geq 0.2\right.$ and $\left.z_{i} \geq 0.2\right)$ are again coincident and concentrated at the corners of the plate, especially at the bottom left and upper right ones, with an optimal number of 6.35 . Table 4 summarizes the optimal positions along with their corresponding coordinates for the convex optimization problem, which provides an objective function evaluation of $h^{*}(w, z, n)=54.2611$. Correspondingly, the Boolean solution using the six actuators and six sensors shown in Table 4 results in $h^{*}\left(\Psi_{a}, \Psi_{s}, n\right)=54.3951$, which again represents a remarkably close approximation to the convex solution.

\subsubsection{Composite panel B2}

Finally, the composite panel with the B geometry is assessed in order to identify the optimal positions for both sensors and actuators. The prior information of the damage coordinates is the same as the one specified in Section 3.3 .1 . while the wave propagation velocity related parameters $a$ and $b$ are defined as in Section 3.2.2. Using 500 samples 


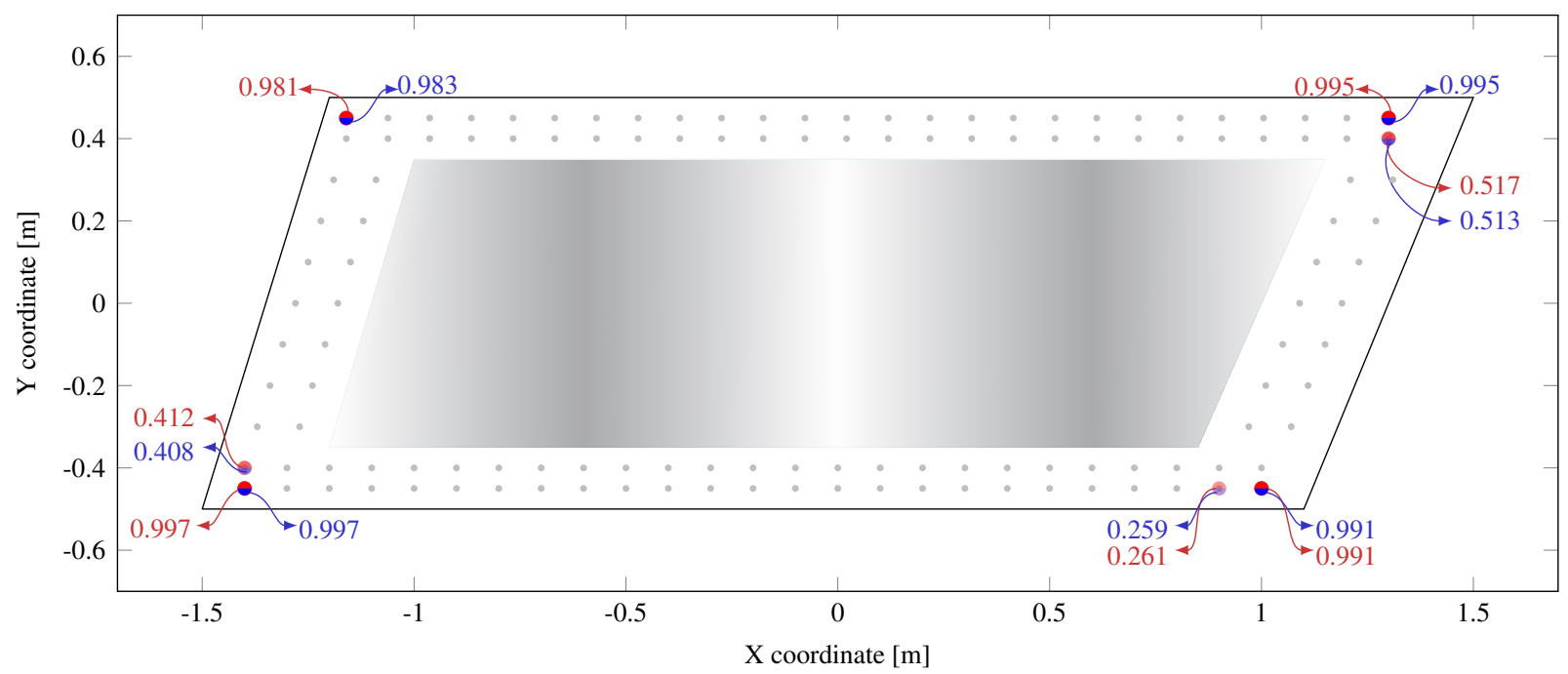

(a) Optimal actuator and sensor configuration for panel B1.

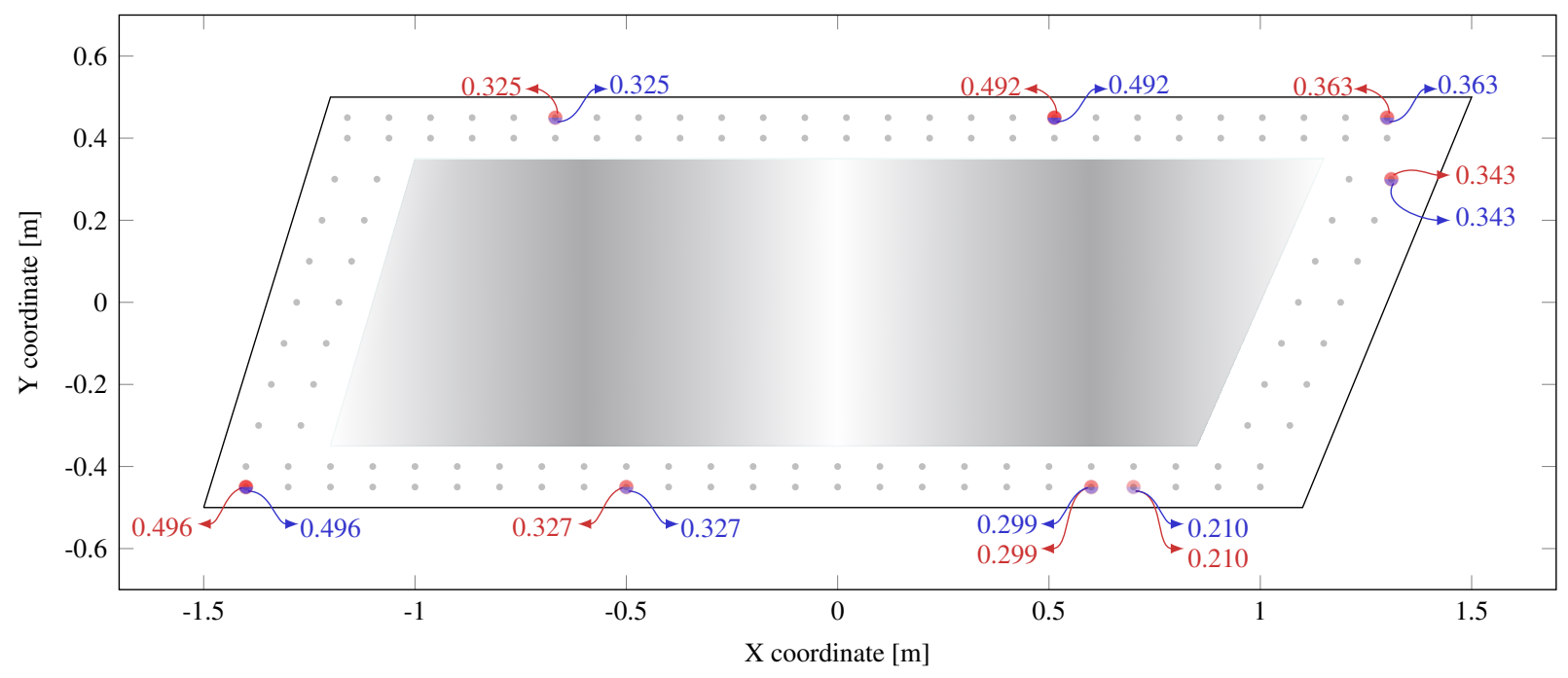

(b) Optimal actuator and sensor configuration for panel B2.

Figure 5: Optimal actuator and sensor configuration for the panel geometry B assuming different materials and prior distributions of the damage occurrence. Panel (a) depicts the case for aluminum alloy 2024-T351 and a bimodal prior distribution of damage occurrence within the gray areas, and (b) shows the case for the composite laminate of layup $\left[-45 / 90_{2} / 45 / 0\right]_{s}$ and the same prior distribution of potential damage occurrence. Red and blue numbers represent the value of actuator and sensor decision variables $\left(w_{j}\right.$ and $\left.z_{i}\right)$, respectively.

of the prior distribution for the evaluation of the objective function, the optimal number of actuators and sensors is $n=10.75$, i.e., 5.37 actuators and 5.37 sensors. Figure $5 \mathrm{~b}$ depicts the optimal layout considering the threshold values of $w_{j} \geq 0.2$ and $z_{i} \geq 0.2$, which distributes both coincident actuators and sensors around the most probable damage occurrence areas. Their corresponding coordinates are also listed in Table 5 . In this case, the evaluation of the objective convex function with the optimal solution provides a value of $h^{*}(w, z, n)=98.2052$. Note that no obvious choice can be made for the Boolean approximation in Table 5 given the small $w$ and $z$ values. Therefore, the approximation is obtained by exploring the combinations of PZT 1 and 6 (i.e. the ones with the largest $w$ and $z$ values) with the 
Table 4: Sensors and actuators above the specified threshold of $w_{j} \geq 0.2$ and $z_{i} \geq 0.2$ along with their corresponding coordinates and the approximated Boolean solution for the panel B1.

\begin{tabular}{lcccccc}
\hline & \multicolumn{2}{c}{ PZT coordinates } & \multicolumn{2}{c}{ Relaxed solution } & \multicolumn{2}{c}{ Binary solution } \\
PZT No. & $X[\mathrm{~m}]$ & $Y[\mathrm{~m}]$ & $w[-]$ & $z[-]$ & $\Psi_{a}$ & $\Psi_{s}$ \\
\hline 1 & -1.400 & -0.400 & 0.412 & 0.408 & 1 & 1 \\
2 & -1.400 & -0.450 & 0.997 & 0.997 & 1 & 1 \\
3 & 0.900 & -0.450 & 0.261 & 0.259 & 0 & 0 \\
4 & 1.000 & -0.450 & 0.991 & 0.991 & 1 & 1 \\
5 & -1.160 & 0.450 & 0.981 & 0.983 & 1 & 1 \\
6 & 1.300 & 0.450 & 0.995 & 0.995 & 1 & 1 \\
7 & 1.300 & 0.400 & 0.517 & 0.513 & & 1 \\
\hline
\end{tabular}

rest of transducers. The best Boolean approximation results to have six sensors and six actuators (defined in Table 5) and leads to $h^{*}\left(\Psi_{a}, \Psi_{s}, n\right)=98.3123$, which is very close to the convex solution. Note also in Figure 5 b that this configuration avoids sensor clustering in PZT 7 and 8 and in PZT 3 and 4 by dropping PZT 3 and 7, respectively.

Table 5: Sensors and actuators above the specified threshold of $w_{j} \geq 0.2$ and $z_{i} \geq 0.2$ along with their corresponding coordinates and the approximated Boolean solution for the panel B2.

\begin{tabular}{lcccccc}
\hline & \multicolumn{2}{c}{ PZT coordinates } & \multicolumn{2}{c}{ Relaxed solution } & \multicolumn{2}{c}{ Binary solution } \\
PZT No. & $X[\mathrm{~m}]$ & $Y[\mathrm{~m}]$ & $w[-]$ & $z[-]$ & $\Psi_{a}$ & $\Psi_{s}$ \\
\hline 1 & -1.400 & -0.450 & 0.496 & 0.496 & 1 & 1 \\
2 & -0.500 & -0.450 & 0.327 & 0.327 & 1 & 1 \\
3 & 0.600 & -0.450 & 0.299 & 0.299 & 0 & 0 \\
4 & 0.700 & -0.450 & 0.210 & 0.210 & 1 & 1 \\
5 & -0.668 & 0.450 & 0.325 & 0.325 & 1 & 1 \\
6 & -0.513 & 0.450 & 0.492 & 0.492 & 0 & 1 \\
7 & 1.300 & 0.450 & 0.363 & 0.363 & 0 & 0 \\
8 & 1.310 & 0.300 & 0.343 & 0.343 & & 1 \\
\hline
\end{tabular}

\section{Discussion}

\subsection{On the case study results}

The proposed methodology for sensor and actuator entropy-based convex optimization has been illustrated for ultrasonic guided-wave based inspection. This optimization problem is addressed by relaxing the position-related decision variables $\left(w_{j}\right.$ and $z_{i}$ ) from binary $\{0,1\}$ to continuous $[0,1]$ values, thus transforming the combinatorial objective function into a convex one (recall Eq. (8)). This relaxation provides a lower bound for the original minimization problem over the binary values. However, the obtained solution cannot be directly translated into an actual optimal sensor and actuator layout when any of these decision variables lie within the open interval $(0,1)$. However, an actual sensor and actuator configuration may be obtained by selecting the positions that have values close to 1 , while avoiding sensor clustering as much as possible. This clustering effect is obtained due to the assumption of the stochastic independence of the predicted data, regardless of how close the positions of the sensor/actuators are [39]. When the optimal convex solution provides relatively low $w$ and $z$ values, the best Boolean approximation is not obvious and it arises from the evaluation of the objective function considering combinations of sensors and actuators with the largest 
$w$ and $z$ values. Note that the use of sub-optimal configurations would lead to more uncertain damage localization with respect to the optimal one.

It is also noticeable from the results that both the location and number of the sensors and actuators are coincident for the aluminum and composite plates and for the different geometries, because they have the same values of $w_{j}$ and $z_{i}$. Since PZT transducers are able to both emit and receive ultrasonic guided-waves, they can act as both a sensor and an actuator and so only half of the PZTs would be needed for the inspection and monitoring of these plate-like structures. Furthermore, note that the resulting optimal PZTs in both aluminum plates are located at the corners of the plate, irrespective of the different prior distributions of damage considered in the case studies (see Figures $4 \mathrm{a}$ and 5a. Given that the aluminum plate provides a homogeneous media for the guided-waves to travel and that the attenuation is not taken into account, placing the PZTs at the corners allows a greater area to be monitored. In the case of the composite structures, the PZTs are spread along the plates with a certain preference for the predominant fiber directions due to the higher wave propagation velocity in such directions. Additionally, observe from the results for the composite panel B2 (Figure 5b) that the optimal PZTs are located around the most probable damage areas of the prior distribution in a triangular pattern.

The optimal number of sensors and actuators is influenced by the type of material, due to the different assumptions adopted in modeling the wave propagation velocity. In particular, the optimal configurations for the aluminum structures contain more sensors with a lower expected information entropy than those obtained for the composite plates. This behavior can be explained by analyzing the objective function in Equation (8), which directly multiplies the expected information entropy by the cost given our choice of the penalty term $\eta=|h(w, z, n)|$, as $h(w, z, n)+|h(w, z, n)| \times s(n)$. To further clarify this behavior, the derivative of the objective function is set to zero so that the optimal number of sensors and actuators can be illustrated in cases where the entropy is different, as follows:

$$
\frac{\partial}{\partial n}\{h(w, z, n)+|h(w, z, n)| \times s(n)\}=0 \Rightarrow \underbrace{\frac{\partial h(w, z, n)}{\partial n} \times\left(1+\frac{h(w, z, n)}{|h(w, z, n)|} s(n)\right)}_{\text {Variation of entropy }}=\underbrace{-\frac{\partial s(n)}{\partial n} \times|h(w, z, n)|}_{\text {Variation of cost }}
$$

Thus, in case of two alternatives with similar $\partial h(w, z, n) / \partial n$ and equal cost $s(n)$, the optimal number of sensors is found to be smaller for structures providing lower variation of entropy. This is depicted in Figure 6, where the derivatives for the aluminum and composite results in panel $\mathrm{A}$ of the case studies are compared. This behavior can be interpreted as a penalty for scenarios with higher uncertainty by reducing the amount of relatively unreliable information. Alternatively, in cases with lower uncertainty (entropy), the quality of such data is higher and more reliable, and hence the proposed approach allows it to use more sensors/actuators.

\subsection{On the ToF model and computational aspects}

The ToF model used in this paper allows the simulation of the time of flight of a scattered ultrasonic guidedwave to reach an arbitrary sensor without the need of a transient ultrasonic guided-wave simulation. This model 


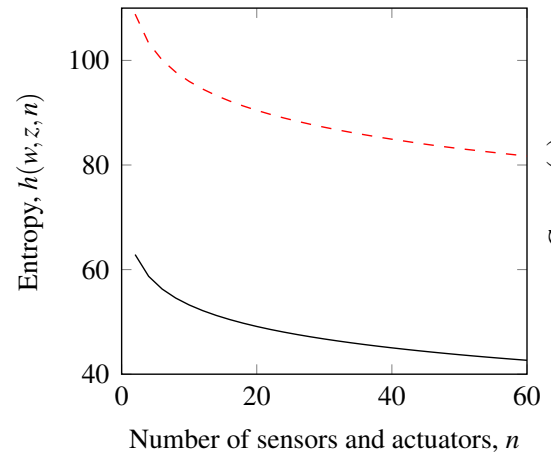

(a) Entropy

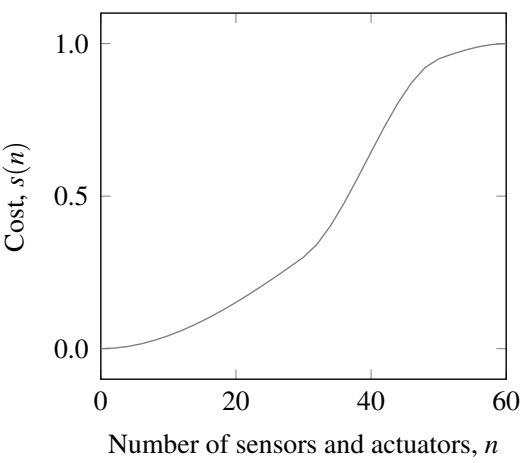

(b) Cost function

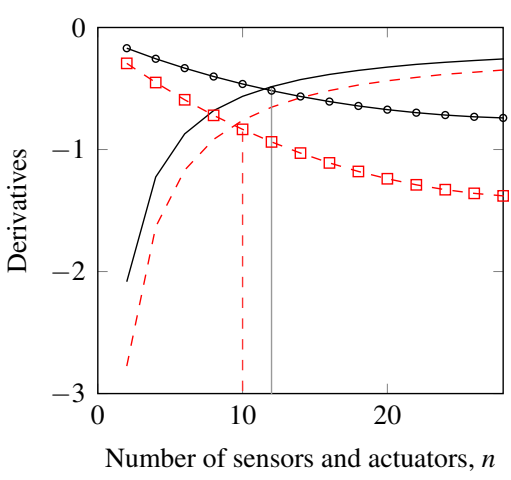

(c) Derivatives and intersection

Figure 6: Behavior of the objective function considering two different entropy curves for optimal distributions of sensors for each $n$, red for the composite and black for the aluminum for panel A of the case studies in (a), the same cost function in (b), and the intersection of the two members (plain lines for variation of entropy, and using markers for the variation of cost) of Eq. (12) in (c).

has proven efficiency when dealing with complex materials and structures, providing the propagation characteristics are known. It is worth mentioning that the elliptical model of the wave propagation velocity used in this paper is only valid for anisotropic structures with quasi-elliptical slowness curves. However, the extension to more complex structures could be achieved by finding mathematical expressions that approximate such slowness curves. In addition, the optimization framework does not account for sensor and actuator malfunctioning, multiple damage scenarios, or the geometry of the structure except for the area of potential damage occurrence and its spatial prior distribution. Note that the robustness of the proposed methodology is expected to be further enhanced should the aforementioned factors be taken into account. An observation from the case studies is that the definition of such prior information is a key aspect, as it can drastically change the optimal sensor layout, at least in the composite structure. Despite its flexibility, the ToF model entails several limitations with regards to the physics of the guided-wave propagation as it does not account for attenuation or wave interaction with different types of damage. Therefore, it is desirable for future work to investigate a physics-based wave propagation model that is continuous and differentiable (e.g. by approximating a finite element model using a surrogate model such as a polynomial chaos expansion [48]), so that the gradients of Equation (5) can be applied.

Nevertheless, the computational efficiency of the proposed approach is remarkable as compared to other approaches in the literature that approximate the optimal binary solution. The optimal designs of the case studies in Section 3 have been obtained in approximately 300 - 400 seconds of runtime (equivalent to 75 - 120 objective function evaluations) in an Intel i3 2-core computer with $8 \mathrm{~Gb}$ of RAM, depending on the type of material and the prior distribution. In contrast, other approaches that use approximation methods such as the forward sequential sensor algorithm [7], take several hours to obtain a suboptimal sensor layout, assuming a fixed distribution of actuators. Therefore, the runtime needed to address the joint search for sensors and actuators would be significantly longer, thus highlighting the benefits of the proposed approach in practice. This efficiency encourages the use of the proposed 
methodology in industrial applications where structures with large and arbitrary geometries and complex materials are used, such as in the aerospace industry. In addition, the use of information-theoretic approaches entail a more robust damage detection that is expected to reduce the number of false alarms and their economic impact.

Finally, note that the proposed method relies on the assumption of a large number of data $N \rightarrow \infty$, so that the posterior distribution can be approximated as Gaussian, ultimately justifying the use of the MAP parameter values [35, 36]. However, this hypothesis causes the model to lose some information about the model error and its uncertainty. Therefore, a more rigorous method would be to use the mutual information between data and model parameters as objective function given by [38, 39]:

$$
I\left(\mathbf{D}_{N}, \boldsymbol{\theta}\right)=H\left(\mathbf{D}_{N}\right)-H\left(\mathbf{D}_{N} \mid \boldsymbol{\theta}\right)
$$

where $H\left(\mathbf{D}_{N}\right)$ is the entropy of the data and $H\left(\mathbf{D}_{N} \mid \boldsymbol{\theta}\right)$ is the conditional entropy. Note that the first term involves a large number of evidence calculations $p\left(\mathbf{D}_{N}\right)$, which is known to be computationally demanding [49] and could make the problem impractical.

\section{Conclusions}

An entropy-based approach for optimal sensor and actuator configuration (number of devices and their position) is proposed in this paper. The methodology exploits the convexity of a relaxed optimization problem where the discrete variables in $\{0,1\}$ are relaxed to continuous variables in $[0,1]$, allowing it to be addressed with standard continuous-variable minimization algorithms at relatively low computational cost. The efficiency and versatility of the optimization method in addressing structures with arbitrary geometries and complex materials is illustrated using two case studies based on ultrasonic guided-wave based inspection. The following conclusions can be drawn:

- The proposed convex optimization method produces a lower bound of the objective function using continuous optimization variables, which are then approximated by Boolean variables to give a near-optimal sensor/actuator configuration. We place the devices at locations of the variables with higher values while avoiding sensor clustering.

- The optimal sensor and actuator layouts coincide, at least for the ultrasonic guided-wave based application, which suggests that the use of PZT transducers in pulse-echo mode is the most efficient test mode.

- The proposed objective function, which combines entropy and cost, provides less sensors/actuators in cases with higher uncertainty (entropy), hence penalizing scenarios with poor quality of data.

Further improvements in our approach are under consideration on the following topics: (1) the inclusion of potential sensor and actuator malfunctioning as well as multiple damage scenarios to further enhance the robustness of the optimal configuration, (2) the computation of the mutual information between data and model parameters by addressing the calculation of the evidence, and (3) the use of a physics-based model, along with a surrogate of it for 
efficient calculations, so that wave interaction with different types of damage as well as wave attenuation are taken into account.

\section{Acknowledgements}

This paper is part of the SAFE-FLY project that has received funding from the European Union's Horizon 2020 research and innovation programme under the Marie Skłodowska-Curie grant agreement No 721455. The authors are also grateful to the California Institute of Technology for kindly hosting the first author.

\section{References}

[1] L. Mujica, M. Ruiz, F. Pozo, J. Rodellar, A. Güemes, A structural damage detection indicator based on principal component analysis and statistical hypothesis testing, Smart Materials and Structures 23 (2) (2013) 025014.

[2] W. Ostachowicz, R. Soman, P. Malinowski, Optimization of sensor placement for structural health monitoring: a review, Structural Health Monitoring 18 (3) (2019) 963-988.

[3] R. A. Howard, Information value theory, IEEE Transactions on Systems Science and Cybernetics 2 (1) (1966) $22-26$.

[4] R. Schlaifer, H. Raiffa, Applied statistical decision theory, MIT Press, 1961.

[5] A. Krause, C. Guestrin, A. Gupta, J. Kleinberg, Near-optimal sensor placements: Maximizing information while minimizing communication cost, in: Proceedings of the 5th International Conference on Information Processing in Sensor Networks, ACM, 2006, pp. 2-10.

[6] G. Capellari, E. Chatzi, S. Mariani, Cost-benefit optimization of structural health monitoring sensor networks, Sensors 18 (7) (2018) 2174.

[7] S. Cantero-Chinchilla, J. Chiachío, M. Chiachío, D. Chronopoulos, A. Jones, Optimal sensor configuration for ultrasonic guided-wave inspection based on value of information, Mechanical Systems and Signal Processing 135 (2020) 106377.

[8] H. Gao, J. Rose, Ultrasonic sensor placement optimization in structural health monitoring using evolutionary strategy, in: AIP Conference Proceedings, Vol. 820, AIP, 2006, pp. 1687-1693.

[9] J. F. Markmiller, F.-K. Chang, Sensor network optimization for a passive sensing impact detection technique, Structural Health Monitoring 9 (1) (2010) 25-39.

[10] M. Thiene, Z. S. Khodaei, M. Aliabadi, Optimal sensor placement for maximum area coverage (MAC) for damage localization in composite structures, Smart Materials and Structures 25 (9) (2016) 095037.

[11] Z. S. Khodaei, M. Aliabadi, An optimization strategy for best sensor placement for damage detection and localization in complex composite structures, in: 8th European Workshop On Structural Health Monitoring (EWSHM 2016), 2016, pp. 5-8.

[12] M. Salmanpour, Z. Sharif Khodaei, M. Aliabadi, Transducer placement optimisation scheme for a delay and sum damage detection algorithm, Structural Control and Health Monitoring 24 (4) (2017) e1898.

[13] H. Tarhini, R. Itani, M. A. Fakih, S. Mustapha, Optimization of piezoelectric wafer placement for structural health-monitoring applications, Journal of Intelligent Material Systems and Structures (2018) 1045389X18799204.

[14] Z. Ismail, S. Mustapha, M. A. Fakih, H. Tarhini, Sensor placement optimization on complex and large metallic and composite structures, Structural Health Monitoring 19 (1) (2020) 262-280.

[15] G. Manson, K. Worden, Lamb wave sensor optimization using differential evolution, in: Smart Structures and Materials 2001: Modeling, Signal Processing, and Control in Smart Structures, Vol. 4326, SPIE, 2001, pp. 570 - 580.

[16] R. Soman, P. Kudela, K. Balasubramaniam, S. K. Singh, P. Malinowski, A study of sensor placement optimization problem for guided wave-based damage detection, Sensors 19 (8) (2019) 1856.

[17] B. Lee, W. Staszewski, Sensor location studies for damage detection with lamb waves, Smart materials and structures 16 (2) (2007) 399.

[18] P. K. Motamed, A. Abedian, M. Nasiri, Optimal sensors layout design based on reference-free damage localization with lamb wave propagation, Structural Control and Health Monitoring (2020) e2490. 
[19] W. J. Staszewski, K. Worden, R. Wardle, G. R. Tomlinson, Fail-safe sensor distributions for impact detection in composite materials, Smart Materials and Structures 9 (3) (2000) 298.

[20] V. Mallardo, M. Aliabadi, Z. S. Khodaei, Optimal sensor positioning for impact localization in smart composite panels, Journal of Intelligent Material Systems and Structures 24 (5) (2013) 559-573.

[21] K. Worden, A. Burrows, Optimal sensor placement for fault detection, Engineering Structures 23 (8) (2001) 885-901.

[22] F. Y. Lin, P.-L. Chiu, A near-optimal sensor placement algorithm to achieve complete coverage-discrimination in sensor networks, IEEE Communications Letters 9 (1) (2005) 43-45.

[23] P. Blanloeuil, N. A. Nurhazli, M. Veidt, Particle swarm optimization for optimal sensor placement in ultrasonic SHM systems, in: Nondestructive Characterization and Monitoring of Advanced Materials, Aerospace, and Civil Infrastructure 2016, Vol. 9804, International Society for Optics and Photonics, 2016, pp. $9804-9804-11$.

[24] Y. Li, X. Wang, R. Huang, Z. Qiu, Actuator placement robust optimization for vibration control system with interval parameters, Aerospace Science and Technology 45 (2015) 88-98.

[25] I. Bruant, L. Gallimard, S. Nikoukar, Optimal piezoelectric actuator and sensor location for active vibration control, using genetic algorithm, Journal of Sound and Vibration 329 (10) (2010) 1615-1635.

[26] V. Gupta, M. Sharma, N. Thakur, Optimization criteria for optimal placement of piezoelectric sensors and actuators on a smart structure: a technical review, Journal of Intelligent Material Systems and Structures 21 (12) (2010) 1227-1243.

[27] S. Boyd, L. Vandenberghe, Convex optimization, Cambridge University Press, 2004.

[28] S. Joshi, S. Boyd, Sensor selection via convex optimization, IEEE Transactions on Signal Processing 57 (2) (2009) $451-462$.

[29] J. A. Taylor, N. Luangsomboon, D. Fooladivanda, Allocating sensors and actuators via optimal estimation and control, IEEE Transactions on Control Systems Technology 25 (3) (2017) 1060-1067.

[30] A. Zare, H. Mohammadi, N. K. Dhingra, T. T. Georgiou, M. R. Jovanovic, Proximal algorithms for large-scale statistical modeling and sensor/actuator selection, IEEE Transactions on Automatic Control (2019) 1-1.

[31] A. Ben-Tal, A. Nemirovski, Lectures on modern convex optimization: analysis, algorithms, and engineering applications, Vol. 2 , Siam, 2001.

[32] D. P. Bertsekas, A. Scientific, Convex optimization algorithms, Athena Scientific Belmont, 2015.

[33] C. Argyris, S. Chowdhury, V. Zabel, C. Papadimitriou, Bayesian optimal sensor placement for crack identification in structures using strain measurements, Structural Control and Health Monitoring 25 (5) (2018) e2137.

[34] G. Capellari, E. Chatzi, S. Mariani, Structural health monitoring sensor network optimization through Bayesian experimental design, ASCEASME Journal of Risk and Uncertainty in Engineering Systems, Part A: Civil Engineering 4 (2) (2018) 04018016.

[35] J. L. Beck, C. Papadimitriou, S.-K. Au, M. W. Vanik, Entropy-based optimal sensor location for structural damage detection, in: Smart Structures and Materials 1998: Smart Systems for Bridges, Structures, and Highways, Vol. 3325, International Society for Optics and Photonics, 1998, pp. 161-173.

[36] C. Papadimitriou, J. L. Beck, S.-K. Au, Entropy-based optimal sensor location for structural model updating, Journal of Vibration and Control 6 (5) (2000) 781-800.

[37] C. Papadimitriou, G. Lombaert, The effect of prediction error correlation on optimal sensor placement in structural dynamics, Mechanical Systems and Signal Processing 28 (2012) 105-127.

[38] P. Bhattacharyya, Optimal sensor placement for Bayesian parametric identification of structures, Ph.D. thesis, California Institute of Technology (2017).

[39] P. Bhattacharyya, J. L. Beck, Exploiting convexification for Bayesian optimal sensor placement by maximization of mutual information, Structural Control and Health Monitoring (2020) (in press).

[40] J. L. Beck, Bayesian system identification based on probability logic, Structural Control and Health Monitoring 17 (7) (2010) $825-847$.

[41] E. T. Jaynes, Information theory and statistical mechanics, Physical Review 106 (4) (1957) 620.

[42] J. L. Beck, L. S. Katafygiotis, Updating models and their uncertainties. i: Bayesian statistical framework, Journal of Engineering Mechanics 124 (4) (1998) 455-461. 
[43] C. Papadimitriou, Optimal sensor placement methodology for parametric identification of structural systems, Journal of Sound and Vibration 278 (4-5) (2004) 923-947.

[44] F. N. Fritsch, R. E. Carlson, Monotone piecewise cubic interpolation, SIAM Journal on Numerical Analysis 17 (2) (1980) $238-246$.

[45] S. Cantero-Chinchilla, J. Chiachío, M. Chiachío, D. Chronopoulos, A. Jones, A robust Bayesian methodology for damage localization in plate-like structures using ultrasonic guided-waves, Mechanical Systems and Signal Processing 122 (2019) 192-205.

[46] C. Fendzi, N. Mechbal, M. Rebillat, M. Guskov, G. Coffignal, A general Bayesian framework for ellipse-based and hyperbola-based damage localization in anisotropic composite plates, Journal of Intelligent Material Systems and Structures 27 (3) (2016) $350-374$.

[47] A. Huber, Dispersion Calculator User's Manual, German Aerospace Center (DLR), Augsburg, Germany (2019).

[48] Y. M. Marzouk, H. N. Najm, Dimensionality reduction and polynomial chaos acceleration of Bayesian inference in inverse problems, Journal of Computational Physics 228 (6) (2009) 1862-1902.

[49] M. Muto, J. L. Beck, Bayesian updating and model class selection for hysteretic structural models using stochastic simulation, Journal of Vibration and Control 14 (1-2) (2008) 7-34. 